\title{
Paramedian Forehead Flap in Post Radiotherapy Fistula
}

\author{
Manish Munjal, Japneet Kaur*, Venus Tilavat, Amanjot Kaur and Shubham Munjal \\ Department of ENT, Dayanand Medical College and Research Institute, Ludhiana, Punjab, India
}

\section{ABSTRACT}

The medial canthal fistula gains notoriety for its unsuccessful " repair " utilising the varied flaps from its vicinity . Ultimately the conventional forehead flap is lone "saviour " in this malady .

Keywords: Paramedian Forehead Flap, Reconstruction, Axial

\section{Introduction}

Maxillofacial fistulae are a surgeon's enigma . Particularly so when the primary malignancy is under control as per clinical and positron emission imaging, evaluation. The acute infraorbital - nasolabial junction, component of the established Weber Ferguson incision is likely to "gape " due to counter forces of traction. A loss of underlying bony support due to removal of the maxilla and the radiation fibrosis effects the facial aesthetics . Primary suturing using advancement flaps, Imeres ' cheek flap or bilobed transposition flaps usually fail to seal the defect .. A median or paramedian flap based on the supra orbital and supra trochlear vessels is the most reliable with the maximum rate of success. Though necessitating a bi staged procedure, the bulk is adequate to obliterate the medial canthal defect . The second stage reposits the redundant flap with trimming if need be, within 4-6 weeks as the case be. The scar at the forehead and the medial canthus is imperceptible at 3-6 months ,subject to individual skin texture .

The forehead flap is one of the oldest recorded surgical techniques for nasal reconstruction. As the gold standard for nasal soft tissue reconstruction, the forehead flap provides a reconstructive surgeon with a robust pedicle and large amount of tissue to reconstruct almost any defect. Modifications provided by masters like Burget and Menick have only increased the utility of this exceptional flap ${ }^{1}$

\section{Case Report}

A 60 yearr old elderly male who had completed a course of fractionated radiotherapy following maxillectomy a year back was taken up for repair of the medial canthal fistula of the left side ( Picture 1). A right side paramedian forehead flap $7.5 \mathrm{~cm}$ in length , $3.5 \mathrm{~cm}$ at base and $1.5 \mathrm{~cm}$ at apex was marked out .

\section{Pedicle planning}

1. The supratrochlear artery was identified at the supraorbital rim using landmarks such as the medial brow border, a point $2 \mathrm{~cm}$ lateral to the midline. (Picture 2)

2. The pedicle was centered over the artery, with a width of $1.5 \mathrm{~cm}$. Smaller pedicles risk damage to the supratrochlear artery. Larger pedicles restrict pivotal movement of the flap.

3. A small amount of extra length was included to allow for flap thickness and swelling. This was done by removing superior standing cutaneous deformity of scalp skin with the distal end of the flap and trimming off during inset.(Picture $3 \& 4$ )

\section{Raise the flap}

1. Local anesthetic was injected circumferentially along the pedicle, inject along the borders only to avoid disrupting the supratrochlear artery.

2. Maked borders of the flap were incised.

3. The flap was raised from distal to proximal (ie. superior to inferior)(picture 3)

4. Layers of the flap-The defect portion of the flap was raised in a subcutaneous plane, leaving the galea/ frontalis down.

$>$ The pedicle was raised in a subgaleal plane, leaving periosteum down, until $1 \mathrm{~cm}$ superior to the level of the eyebrow. It was then transitioned down to a subperiostial plane to keep the supratrochlear artery protected.

$>$ When the proximal end of the flap was extended across the orbital rim an extra $1.5 \mathrm{~cm}$ of length was obtained. The artery was sandwiched between the corrugator and frontalis muscles in this area 


\section{Close the donor defect}

1. Undermining is done in a subgaleal plane and deep sutures should be placed through galeal layer to ensure adequate strength.(Picture 5)

2. Suture: interrupted 3-0 PDS, 3-0 vicryl, or 3-0 monocryl for subcutaneous tissues, 5-0 black nylon vertical mattress for skin.(Picture 6)

3. The backside of the flap may be covered with thin Alloderm or a split thickness skin graft as a biological dressing and to decrease oozing.

\section{Flap inset}

1. The flap was pivoted clockwise to reach the defect. Rotation was done so that the flap skin is facing the ipsilateral eye can decrease ooze from the backend

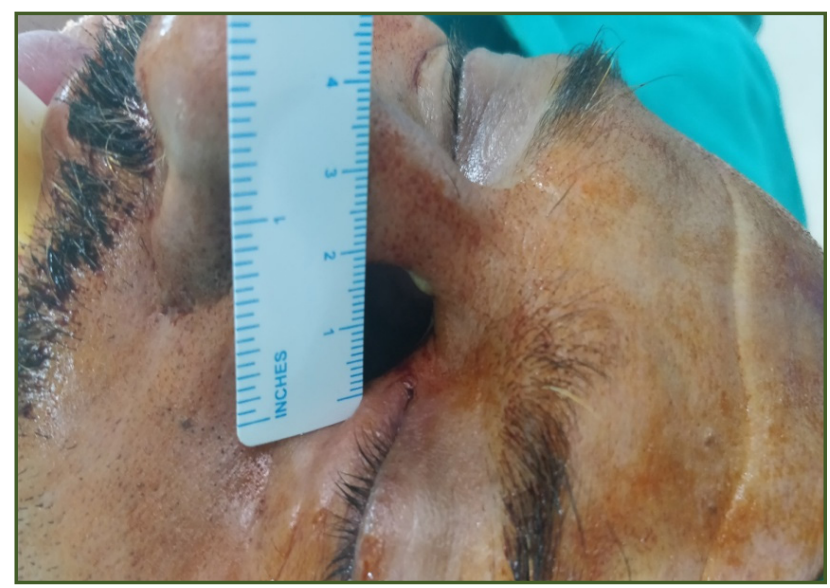

Picture 1A

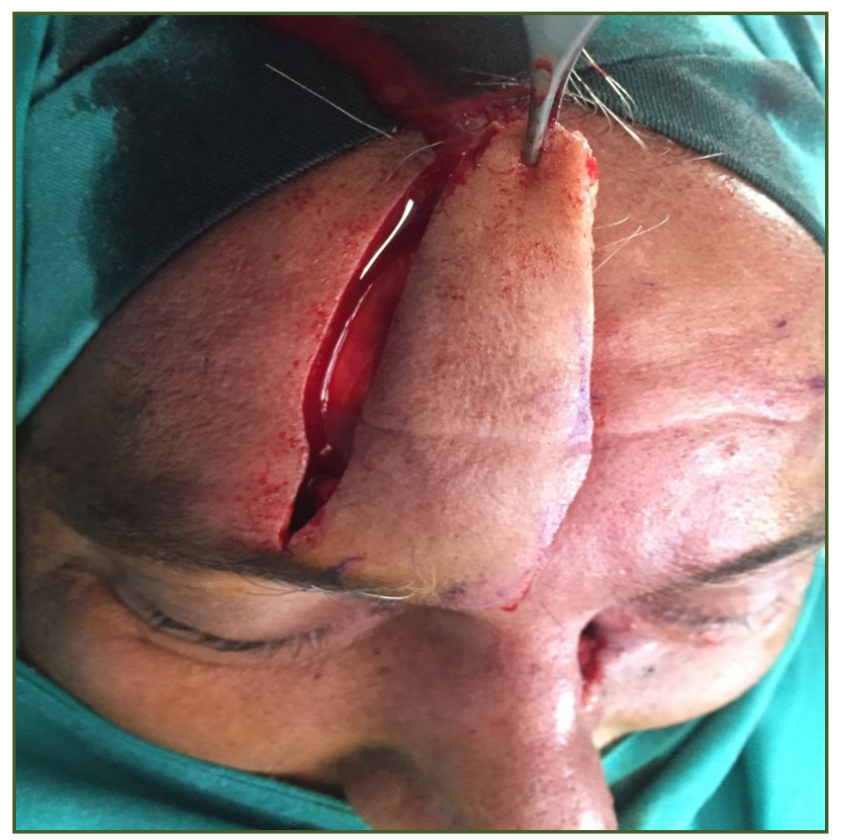

Picture 3

Annals of Applied Bio-Sciences, Vol. 4; Issue 4: 2017 of the flap from reaching the eye. Undermining of adjacent defect skin was done to decrease trap door defect.(picture 7)

2. Suture: 5-0 or 6-0 nylon/prolene for skin only. No deep sutures. A minimum of sutures are used so as to minimize risk for flap necrosis(Picture 8 \&9)

On seventh postoperative day, the sutures were removed. The patient was scheduled for flap detachment approximately 3 weeks from the date of initial surgery. Pedicle separation was done with the patient under local anesthesia. The pedicle was separated sharply, base of the pedicle is returned to the glabellar region to achieve a normal intereyebrow distance.

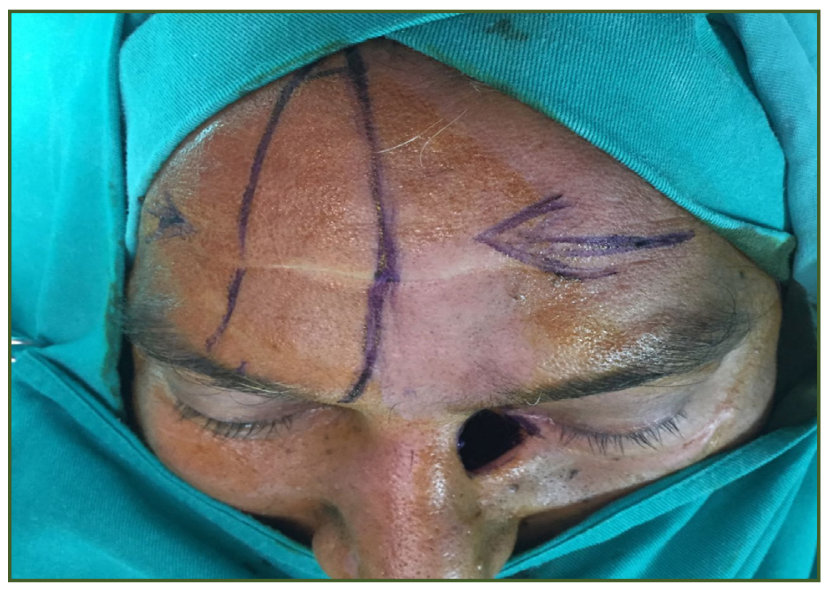

Picture 1B

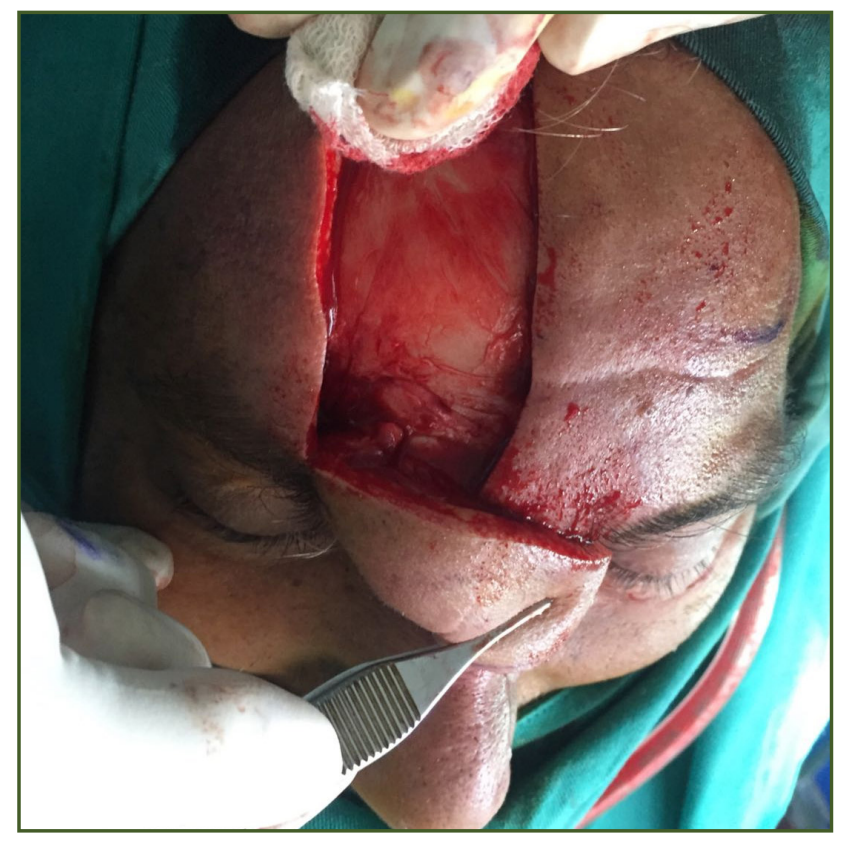

Picture 4 


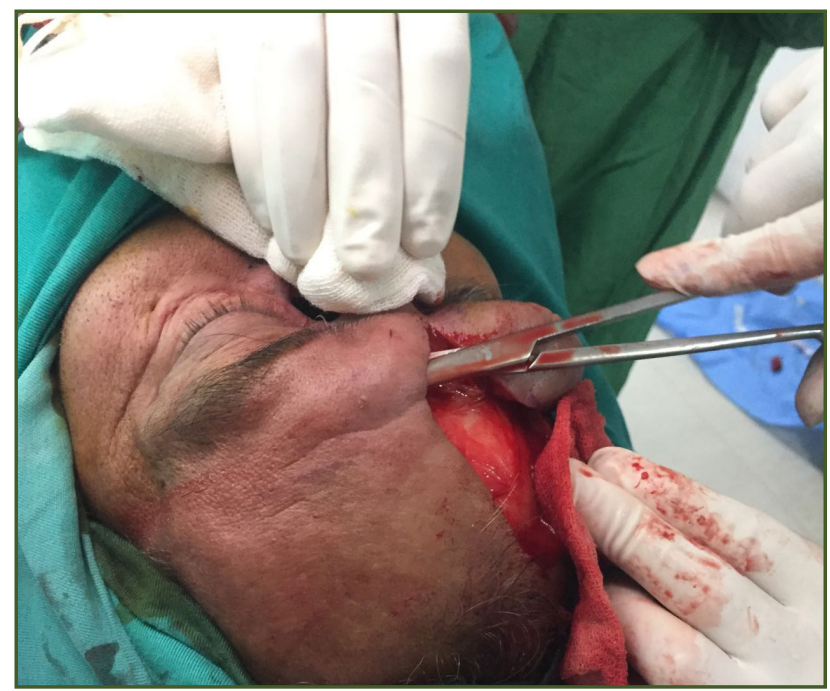

Picture 5

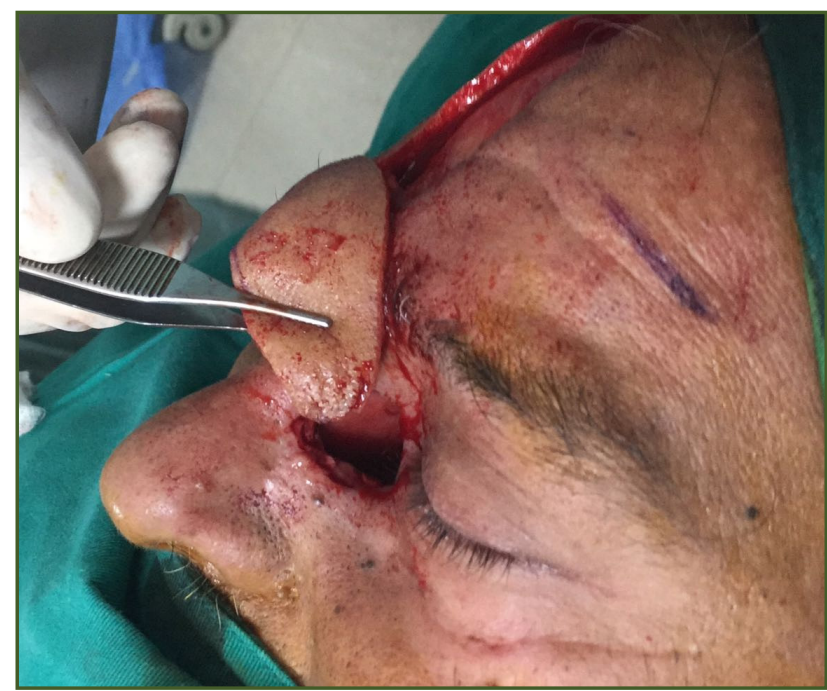

Picture 7

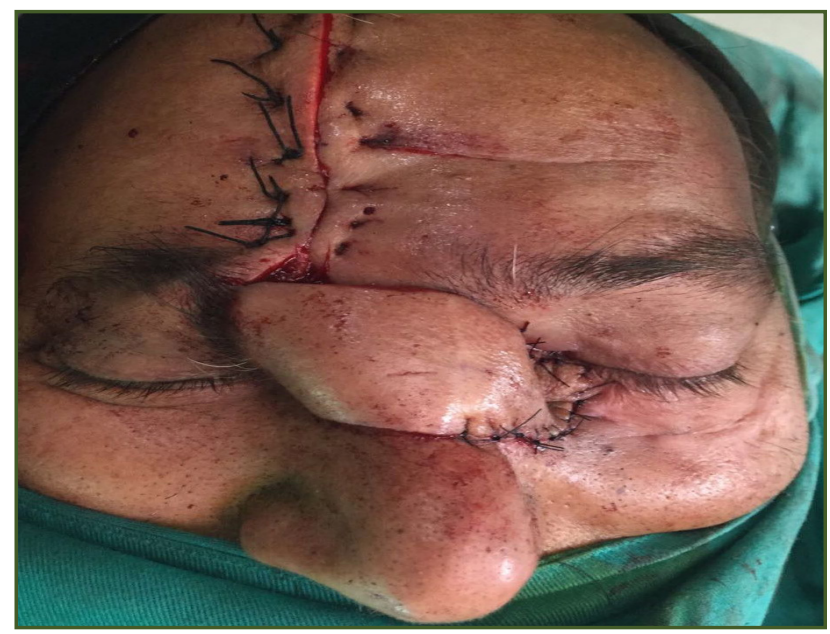

Picture 9

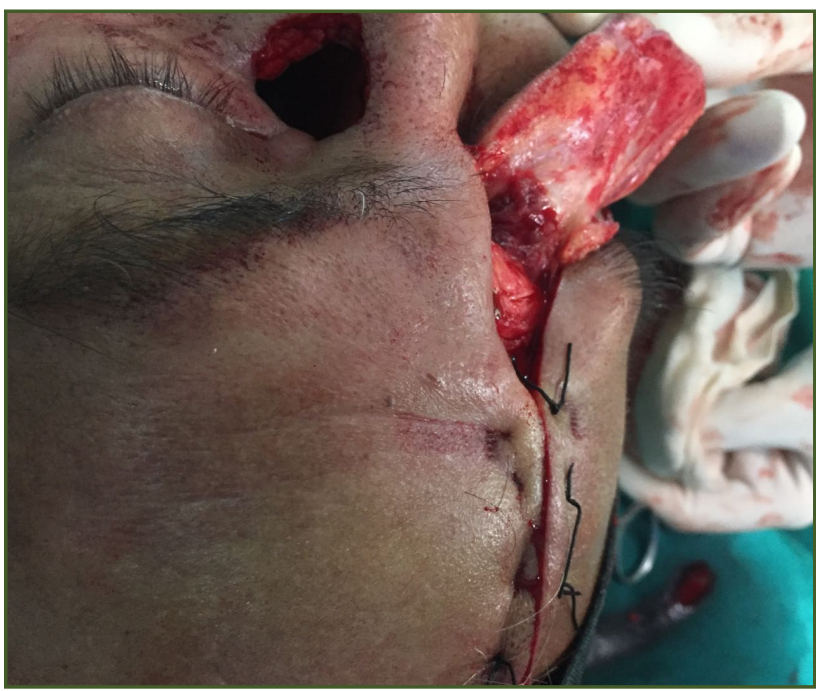

Picture 6

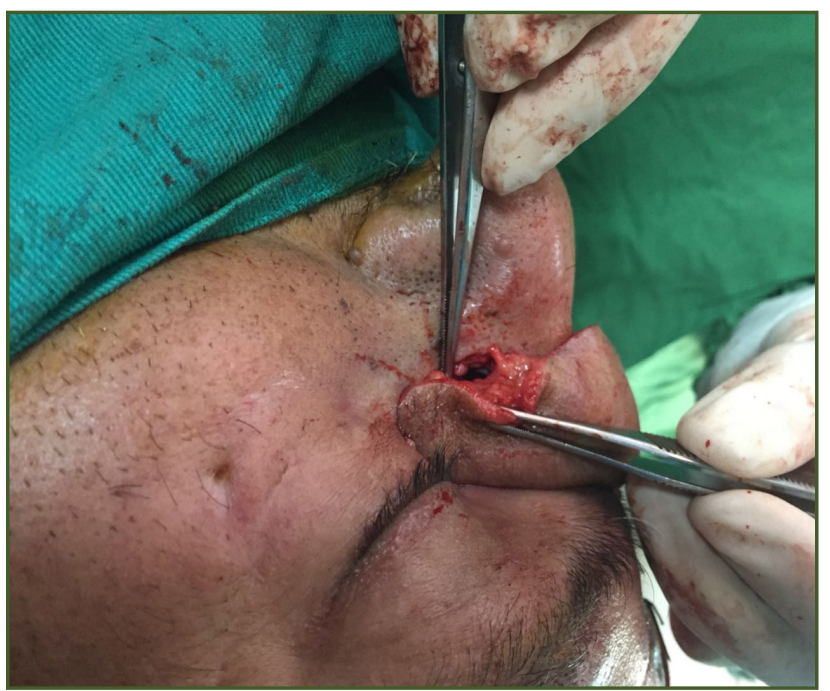

Picture 8

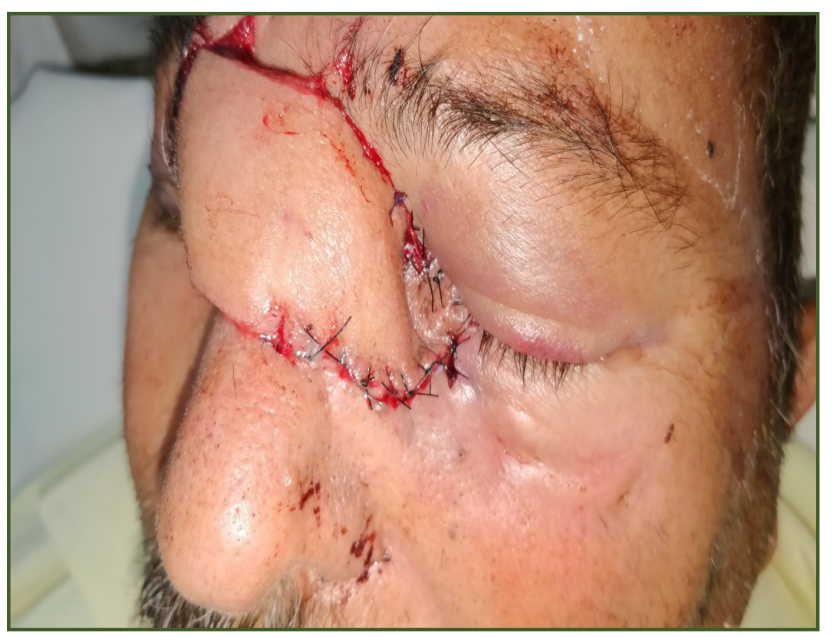

Picture 10 


\section{Discussion}

The tint of forehead skin so exactly matches that of the face and nose that it must be first choice. Is not the forehead the crowning feature of the face and important in expression? Why then should we jeopardize its beauty to make a nose? First, because in many instances, the forehead makes far and away the best nose. Second, with some plastic juggling, the forehead defect can be camouflaged effectively." - Sir Harold Gillies and D. Ralph Millard²

The forehead is acknowledged as the preferred donor site for resurfacing the nose. Small defects less than $1.5 \mathrm{~cm}$ can be with local flaps. Superficial defects consisting of skin and minimal subcutaneous fat can be repaired with skin grafts. Limited alar defects can be successfully repaired with a nasolabial flap. The forehead flap, however, remains the premier donor site for a nasal reconstruction because of its size, vascularity, and excellent color, texture, and skin thinness. A paramedian forehead flap allows the transfer of forehead tissue in an efficient and reliable manner with minimal donor deformity and creates the most aesthetically pleasing reconstruction, both to the recipient nose and the donor forehead. Although there has been a shift away from midline forehead tissues toward more extensive or less satisfactory methods such as the scalping flap, skin expansion, or the use of distant or free flaps in nasal reconstruction, the paramedian forehead flap remains the best choice $^{3}$

The paramedian forehead flap is based on the supratrochlear artery .The supratrochlear artery was precisely located $1.7-2.2 \mathrm{~cm}$ from the midline, corresponding to the medial border of the eyebrow. The notch of the supratrochlear vessels is palpable and that is where the skin pedicle was centered.(Picture 2). The supratrochlear artery and the supraorbital artery, which lie laterally, are terminal branches of the ophthalmic artery (branch of the internal carotid artery), and supplies the anterior pericranium and galea.The supratrochlear artery divides into superficial and deep branches. The superficial branch enters the frontalis muscle and runs on the surface of the galea until entering the subcutaneous tissue approximately $3.5 \mathrm{~cm}$ above the orbital rim. The deep branch runs within the subgaleal fascia (this layer may be considered a component of the pericranium). The deep branches pursue an axial course for approximately 1.5 to $4 \mathrm{~cm}$ above the supraorbital rim. There are many penetrating vessels that connect the superficial and deep branches. These vessels are divided if the galea is separated from the underlying pericranium. The pedicle base is perfused by a terminal branch of the angular artery that is actually a major contributor to the flap's arterial supply.

One disadvantage of the paramedian forehead flap is the vertical forehead scar. The wound edges must be carefully approximated to minimize wound tension. For large secondary defects, we achieve this by widely undermining the forehead skin, to the temporalis muscle bilaterally. Additional length can be achieved by performing galeatomies on either side of the incision. Inability to achieve primary closure is not a contraindication to performing a forehead flap. Allowing the donor site to heal in part by secondary intention usually results in an acceptable cosmetic result. Another disadvantage of the paramedian forehead flap is its limited length in patients with low hairlines. Several modifications are possible, such as the oblique forehead flap, tissue expansion, or extension of the flap into hair-bearing scalp. ${ }^{4}$

\section{Conclusion}

A paramedian forehead flap allows the transfer of forehead tissue in an efficient and reliable manner with minimal donor deformity and creates the most aesthetically pleasing reconstruction, both to the recipient defect and the donor forehead. The surgeon should clearly plan the flap and defect dimensions in order to obtain good results.

\section{Reference}

1. Correa BJ, Weathers WM, Wolfswinkel EM, Thornton JF. The Forehead Flap: The Gold Standard of Nasal Soft Tissue Reconstruction. Seminars in Plastic Surgery. 2013;27(2):96103. doi:10.1055/s-0033-1351231.

2. Gillies, H., and Millard, R. The Principles and Art of Plastic Surgery. Boston: Little, Brown, 1957.

3. Menick FJ. A ten-year experience in nasal reconstruction with the three-stage forehead flap. Plast. Reconstr. Surg. 109: 1839, 2002

4. Hoffman HT, Baker SR. Nasal reconstruction with the rapidly expanded forehead flap. Laryngoscope. 1989;99;1096- 1098

*Corresponding author:

Dr Japneet Kaur, Department of ENT, Dayanand Medical College and Research Institute, Ludhiana, Punjab, India

Phone: +91 09902549237

Email: drjapneetkaur@gmail.com

Financial or other Competing Interests: None.

Date of Submission : 04.12.2017

Date of Acceptance : 15.12.2017

Date of Publication : 24.12.2017 